\title{
THE IMPACT OF MACULAR DISEASE ON PEDESTRIAN DETECTION: A DRIVING SIMULATOR EVALUATION
}

\author{
P. Matt Bronstad, Alex R. Bowers, Robert B. Goldstein, Amanda Albu, Eli Peli \\ The Schepens Eye Research Institute \\ Department of Ophthalmology \\ Harvard Medical School \\ Boston, Massachusetts, USA \\ Email: matthew.bronstad@schepens.harvard.edu
}

Summary: We describe the design of a driving simulator study to determine the effect of central visual field loss (due to macular disease) on pedestrian detection when driving. Pilot data suggest that a scotoma (blind area) in the central visual field can impair driving by increasing response time to hazardous circumstances.

\section{INTRODUCTION}

Regulations concerning the minimum vision requirements for driver licensing vary widely across states (Peli and Peli, 2002). For an unrestricted driving license, minimum visual acuity varies from $20 / 40$ to $20 / 70$, while for a restricted license (e.g., daylight driving only), it varies from $20 / 40$ to 20/200 (although some states do not specify an absolute minimum). There is a shortage of data regarding the impact of impaired vision on driving behaviors, which probably contributes to the varying requirements. Age-related macular degeneration is a major cause of irreversible vision loss affecting 1.75 million Americans aged over 40 years (Friedman et al., 2004). Macular degeneration and other conditions (optic atrophy, juvenile macular dystrophies, and diabetic maculopathy) impair central vision causing a reduction in visual acuity and blind areas (scotomas) in the central visual field, although peripheral vision is usually normal. Surprisingly, almost all regulations treat central vision impairment simply as a visual acuity loss with no consideration given to the impact of central field loss. Peripheral field loss is a consideration in driving licensing in most jurisdictions, but central field loss is never or rarely considered.

Both peripheral and central field loss could be hazardous for driving; objects that fall within the area of field loss might go undetected until it is too late to respond safely. Unlike the typical visual field measurement situation when eyes are stationary, in the real world eye scanning will move the area of field loss around so that the obscured area of the scene changes over time. Recent on-road studies have demonstrated that even mild to moderate peripheral field loss results in measurable decrements in driving performance, though most participants were still considered safe drivers (Bowers et al., 2005; Haymes et al., 2008). In on-road studies evaluating drivers with hemianopia (a more severe field loss involving half the visual field on one side in both eyes), between $30 \%$ and $80 \%$ of participants failed the driving test (Tant et al., 2002; Tant, 2008; Wood et al., 2008). In a driving simulator study, drivers with hemianopia had significantly poorer detection rates for pedestrians on the side of their visual loss than on their unaffected side, despite their attempts to compensate by scanning toward the non-seeing side of the field (Bowers et al., 2007; Bowers et al., 2008). 
In many states, people with central field loss are permitted to drive with a restricted license (Peli and Peli, 2002). To our knowledge, however, no study has specifically addressed the effects of central field loss on driving. In the present study we are investigating the impact of central field loss on pedestrian detection and driving behaviors. Many people with central field loss view eccentrically using an extrafoveal location: the preferred retinal locus; the retinal area used for fixation, reading, and other visual tasks instead of the damaged fovea (Timberlake et al., 1986). The preferred retinal locus is usually adjacent to the scotoma and its location varies from person to person (Fletcher and Schuchard, 1997). Whereas in juvenile macular degeneration the preferred retinal locus is usually below the scotoma (Sunness et al., 1996), in age-related macular degeneration the preferred retinal locus is more frequently lateral to the scotoma (Guez et al., 1993; Sunness et al., 1996). As illustrated in Fig 1, a preferred retinal locus lateral to the scotoma may obscure important driving-relevant information on one side of the road while a preferred retinal locus below the scotoma may keep most driving-relevant information in the field of view (except possibly for traffic lights and road signs).

A pedestrian moving on a collision course with a vehicle will remain in the same part of the driver's visual field if the driver looks straight down the road (see Fig 1a). Figure 1 also shows how the same situation may appear to a driver with a $10^{\circ}$ lateral scotoma (B), and to a driver with a vertical scotoma $(\mathrm{C})$. The pedestrian may remain invisible to the driver with the lateral scotoma (1B) long enough to engender a dangerous situation. This potential hazard cannot be predicted from the driver's visual acuity. A person with central field loss with the same visual acuity but with a preferred retinal locus below their scotoma will not have the same difficulty (1C).
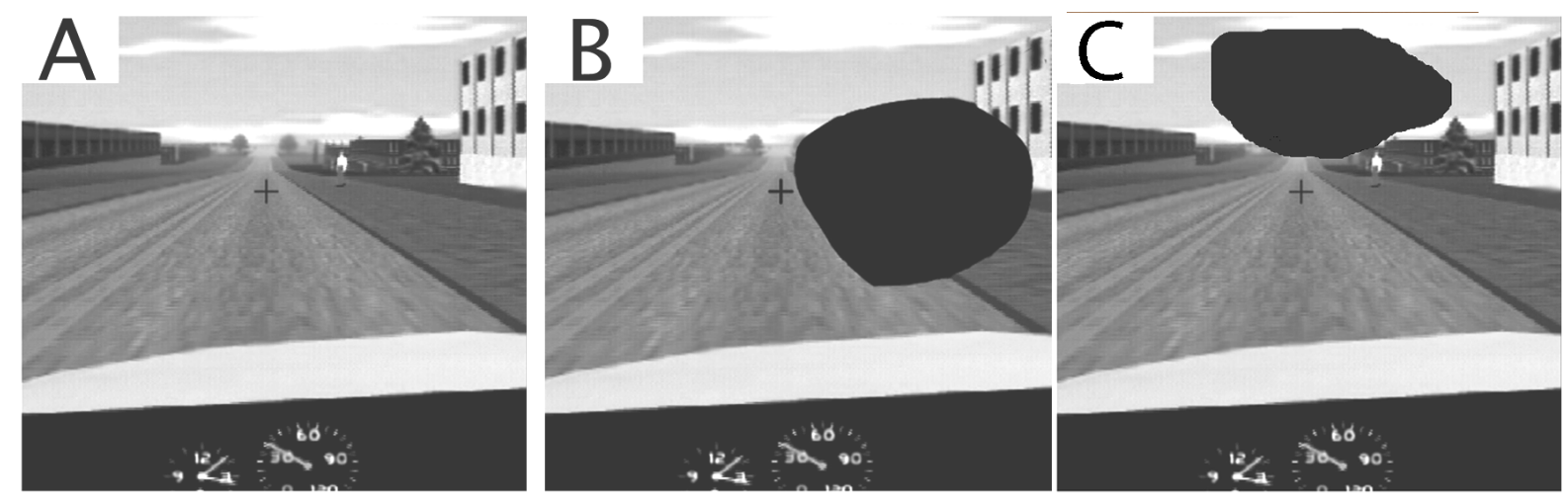

Figure 1. Road appearance under three different vision conditions: (A) Normal field of vision; (B) Lateral scotoma; (C) Vertical scotoma.

On the other hand, people with lateral scotoma may scan frequently to compensate for their visual loss. To test whether lateral scotomas affect responsiveness in collision situations we used a driving simulator and implemented a set of driving scenarios with pedestrians walking or running to maintain collision courses with the approaching drivers. We programmed the virtual pedestrians so that they would maintain a relatively constant eccentricity with respect to the subject's vehicle, and thus remain on a collision course. In this paper we report preliminary data from pilot subjects. 


\section{METHODS}

\section{Driving Simulator}

We use a high-fidelity PP-1000 driving simulator (FAAC, Inc., Ann Arbor, MI). The simulator has $225^{\circ}$ horizontal field of view, a rumble seat, and standard driving controls for a car with automatic transmission (Peli et al., 2005). The cab interior resembles a Crown Victoria.

\section{Driving Scenarios}

For the pilot subjects reported in this paper, we used 3 drives in a city environment $(48 \mathrm{kph} / 30$ $\mathrm{mph}$ ) and 2 drives on a rural highway $(97 \mathrm{kph} / 60 \mathrm{mph})$. Each drive lasted 6-12 minutes depending on the subject's speed. There were 12-14 pedestrian appearances in each scenario. The initial appearance locations were at $-14^{\circ},-4^{\circ}, 4^{\circ}$, and $14^{\circ}$ eccentricities, at $67 \mathrm{~m}\left(220^{\prime}\right)$ (city) and $134 \mathrm{~m}\left(440^{\prime}\right)$ (highway) from the participant's vehicle. These distances correspond to approximately 5 seconds before collision at the posted speeds of $30 \mathrm{mph}$ and $60 \mathrm{mph}$, respectively. After appearing, the pedestrians walked or ran towards the road on a collision course with the approaching driver, maintaining a relatively constant eccentricity with respect to the driver; however, they stopped before a collision occurred. The small eccentricity represented a hazard approaching from the next travel lane or the sidewalk beside the subject's travel lane, while the larger eccentricity represented a hazard approaching at a faster speed from a greater distance (e.g, the far sidewalk).

People with central field loss who could potentially be driving (e.g., permitted to drive in some states with a restricted license) will typically have visual acuity of 20/200 or better (Peli and Peli, 2002). At that acuity level, the central scotoma is unlikely to be greater than $10^{\circ}$ in diameter. In the case of a scotoma lateral to the preferred retinal locus, pedestrians at smaller eccentricities on the scotoma side are likely to be within the scotoma, while pedestrians at larger eccentricities are unlikely to be obscured by the scotoma (Figs $1 \mathrm{~b}$ and 2). Thus the $4^{\circ}$ and $14^{\circ}$ eccentricities enable us to test the following hypotheses about the detection performance of people with lateral scotomas (preferred retinal locus to the left or right of the scotoma): (1) detection will be more impaired for pedestrians at the smaller than the larger eccentricity on the scotoma side; and (2) detection will be less impaired at the smaller eccentricity on the scotoma side than for pedestrians on the non-scotoma side. Conversely we expect no lateralized differences in detection performance for people with vertical scotomas (preferred retinal locus below scotoma).

Figure 2, derived from 4 drivers with central field loss, shows for the 4 pedestrian placement locations, the initial location of pedestrians in the visual field (smaller boxes) and their probable location 3 seconds later (larger boxes), assuming a vehicle speed of approximately $30 \mathrm{mph}$ (city) or $60 \mathrm{mph}$ (highway) and that the subject looked directly down the roadway. Despite our best efforts, it is difficult to ensure the pedestrian placement is perfect; it depends on uncontrollable factors such as the driver's exact lateral position within the lane, speed, and heading. Placement is also affected by constraints in the virtual world that may result in small shifts of pedestrian locations, such as presence of foliage. 


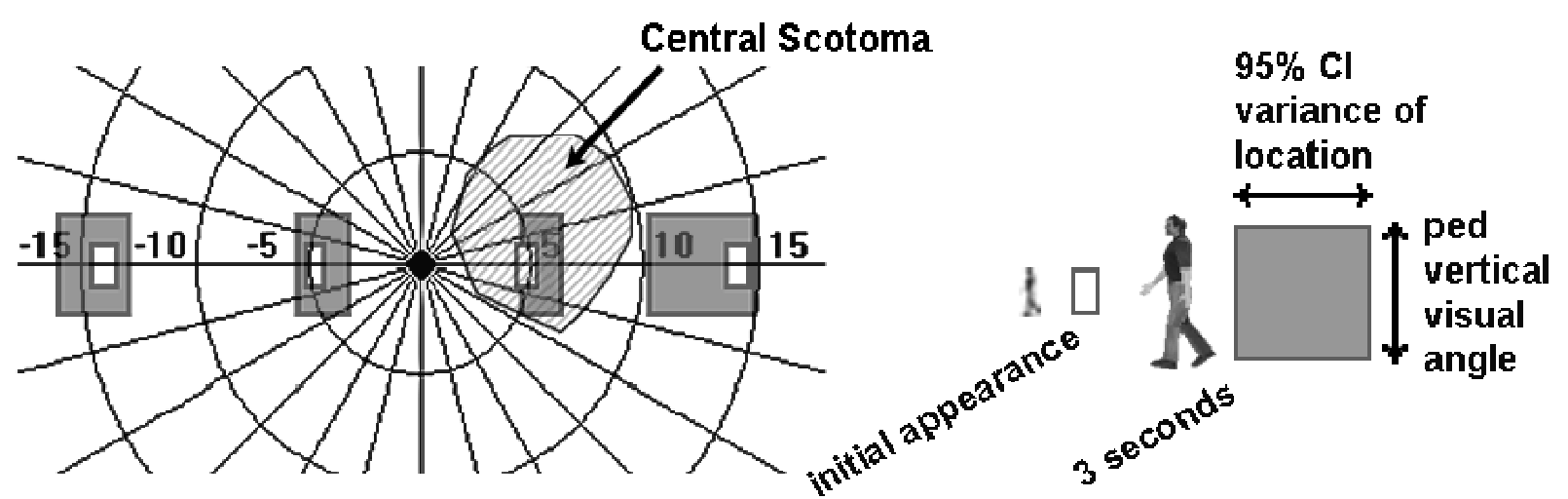

Figure 2. Pedestrian placement projected onto a hypothetical visual field plot. In this case a patient with a lateral preferred retinal locus to the left of the scotoma will tend to miss pedestrians at the $4^{\circ}$ right eccentricity throughout the first 3 sec but not pedestrians at the $14^{\circ}$ right eccentricity.

\section{Procedure}

Subjects made two visits, the first for visual assessment, and the second for the driving simulator session. We took $30-60$ minutes at the start of the simulator session to acclimate subjects to driving in the virtual environment and to practicing the pedestrian detection task. We implemented a variety of precautions to reduce simulator sickness.

Participants were instructed to press the horn as soon as they saw a pedestrian appear; these honks were analyzed to determine how many targets were detected, at what particular eccentricities, and for reaction latency. We used these measures to determine relationships between scotoma location and detection of pedestrian targets in corresponding areas of the visual field.

\section{Analyses}

We used nonparametric statistics (Mann-Whitney) to test the hypothesis that lateral scotomas would impair detection of pedestrians that appeared at locations likely to be covered by the scotoma.

\section{PRELIMINARY RESULTS}

Here we report data from 4 pilot subjects, all men who had stopped driving within the last 7 to 20 years. Binocular visual field plots are shown in the top row of Fig 3; reaction times and detection rates are in the bottom row. While scotoma location did not impact detection rates (detection rates of all subjects were close to $100 \%$ at all eccentricities), it significantly affected reaction times. The first two subjects (A \& B) have lateral right scotomas. Their reaction times for the pedestrians at $4^{\circ}$ eccentricity were significantly longer than both their reaction time for the pedestrians that appeared at $14^{\circ}$ eccentricity to the right and that of pedestrians appearing at $4^{\circ}$ eccentricity to the left. The two drivers with vertical scotomas (C\&D) showed no differences in reaction times to pedestrians on the right and left. 
(A1) Pilot 1 - Lateral right scotoma Age 75. Visual Acuity (VA) 20/60

11 years since disease onset

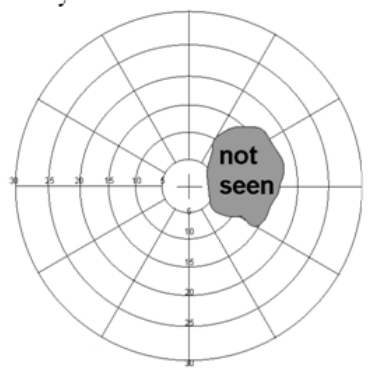

(A2)

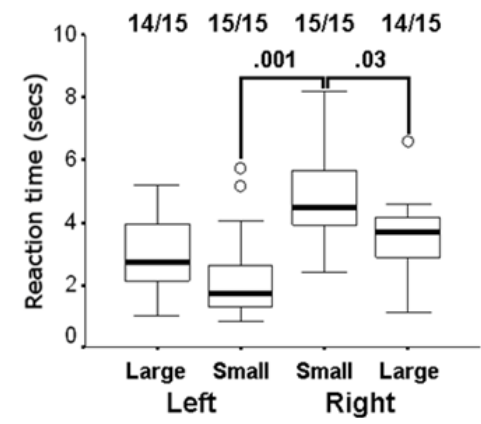

(B1)

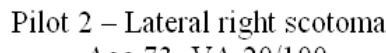
Age 73. VA 20/100

7 years since disease onset

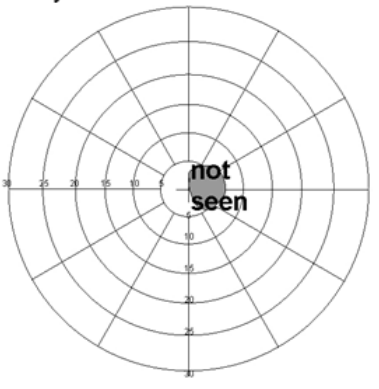

(B2)

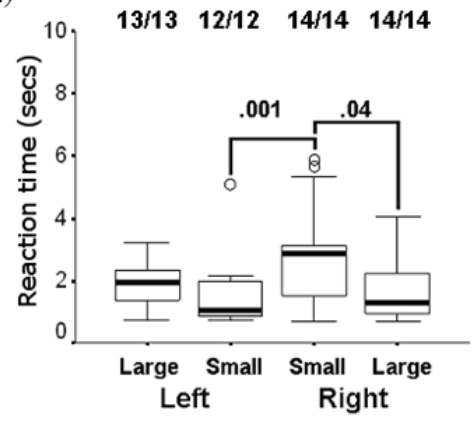

(C1)

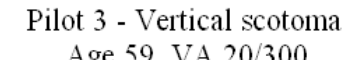

Age 59. VA 20/300

23 years since disease onset

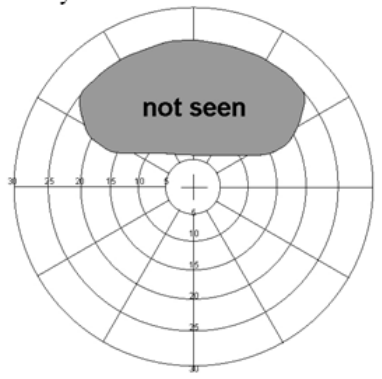

(C2)

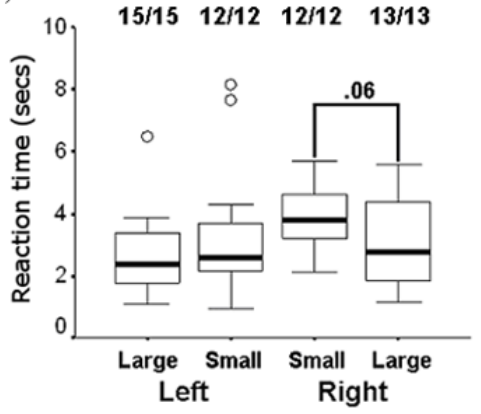

(D1)

Pilot 4 - Vertical scotoma Age 63. VA 20/80

11 years since disease onset

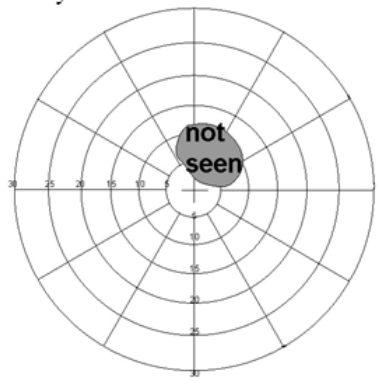

(D2)

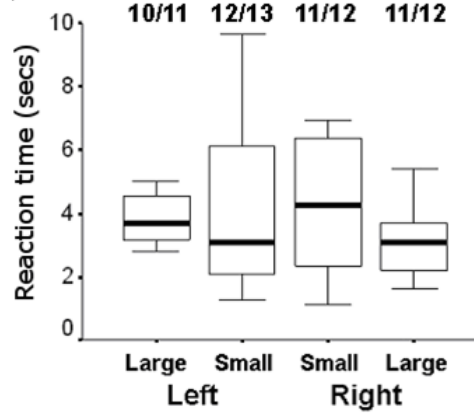

Figure 3. Pilot data from 4 subjects. Top: Binocular visual field plots. Bottom: reaction time medians and interquartile ranges (IQR) for large (14 ${ }^{\circ}$ and small $\left(4^{\circ}\right)$ eccentricities. Detection rates (targets seen/total) are above each boxplot. P-values indicate RT differences that are significantly different or marginal. Whiskers represent largest non-outlier data within 1.5x IQR. Circles represent outliers. 


\section{DISCUSSION}

Preliminary analyses provide initial confirmation of our main hypothesis that a scotoma lateral to fixation in the central visual field affects pedestrian detection more so than a vertical scotoma. Even with a small sample of pilot data, the two participants with lateral scotomas had significantly longer detection times (but similar detection rates) for pedestrians that were likely to fall in their scotoma than pedestrians that were unlikely to be obscured by their scotoma. By comparison, the participants with vertical scotomas did not exhibit any significant differences between reaction times at any location.

We are continuing to collect data from drivers with central field loss and matching them to normally-sighted drivers. For both groups we will include drivers with more recent driving experience. Group data will be reported in a subsequent paper.

\section{ACKNOWLEDGEMENTS}

This research is supported by NIH grants EY12890 (EP) and K99 EY018680 (ARB). We thank Vincent Ciaccio and Aaron Mandel for help in scenario testing and development. Driving simulator facilities were provided by Dr. Joseph Rizzo of the Center for Innovative Visual Rehabilitation at the Boston Veterans Administration Hospital.

\section{REFERENCES}

Bowers, A., Mandel, A., Goldstein, B. and Peli, E. (2008). Driving with hemianopia: Head scanning and detection performance in a simulator. Investigative Ophthalmology and Visual Science 49, ARVO E-Abstract 4105.

Bowers, A., Peli, E., Elgin, J., McGwin, G. and Owsley, C. (2005). On-road driving with moderate visual field loss. Optom Vis Sci 82, 657-667.

Bowers, A. R., Mandel, A. J., Goldstein, B. G. and Peli, E. (2007). Simulator-based driving with hemianopia: detection performance and compensatory behaviors on approach to intersections. Proceedings of the 4th International Driving Symposium on Human Factors in Driver Assessment, Training, and Vehicle Design, Stevenson, WA. CD-ROM 269-270.

Fletcher, D. C. and Schuchard, R. A. (1997). Preferred retinal loci relationship to macular scotomas in a low-vision population. Ophthalmol 104, 632-638.

Friedman, D. S., O'Colmain, B., Tomany, S. C., McCarty, C., de Jong, P., Nemesure, B., Mitchell, P., Kempen, J. and Congdon, N. (2004). Prevalence of age-related macular degeneration in the United States. Arch Ophthalmol 122, 564-572.

Guez, J.-E., Le Gargasson, J.-F., Rigaudiere, F. and O'Regan, J. K. (1993). Is there a systematic location for the pseudo-fovea in patients with central scotoma? Vision Research 33, 12711279.

Haymes, S. A., LeBlanc, R. P., Nicolela, M. T., Chiasson, L. A. and Chauhan, B. C. (2008). Glaucoma and on-road driving performance. Invest. Ophthalmol. Vis. Sci. 49, 3035-3041. 
Peli, E., Bowers, A. R., Mandel, A. J., Higgins, K. E., Goldstein, R. B. and Bobrow, L. (2005). Design of driving simulator performance evaluations for driving with vision impairments and visual aids. Transportation Research Record 1937, 128-135.

Peli, E. and Peli, D. (2002). Driving with Confidence: A Practical Guide to Driving with Low Vision., World Scientific Publishing Co. Pte. Ltd, Singapore.

Sunness, J. S., Applegate, C. A., Haselwood, D. and Rubin, G. S. (1996). Fixation patterns and reading rates in eyes with central scotomas from advanced atrophic age-related macular degeneration and Stargardt disease. Ophthalmol 103, 1458-1466.

Tant, M. (2008). Do peripheral prism visual field expansion glasses assist drivers with hemianopia? 9th International Conference on Low Vision, Vision 2008, Montreal, Canada, CD-ROM Abstract 2.2.

Tant, M. L. M., Brouwer, W. H., Cornelissen, F. W. and Kooijman, A. C. (2002). Driving and visuospatial performance in people with hemianopia. Neuropsychological Rehabilitation 12, 419-437.

Timberlake, G. T., Mainster, M. A., Peli, E., Augliere, R. A., Essock, E. A. and Arend, L. E. (1986). Reading with a macular scotoma. I. Retinal location of scotoma and fixation area. Invest Ophthalmol Vis Sci 27, 1137-47.

Wood, J. M., Elgin, J., McGwin Jr., G., Meek, G. C., Vaphiades, M. S., Braswell, R. A., DeCarlo, D. K., Kline, L. B., Brenner, D. A. and Owsley, C. (2008). On-road driving performance by persons with hemianopia and quadrantanopia. Invest Ophthalmol Vis Sci 49, ARVO E-abstract 3155. 